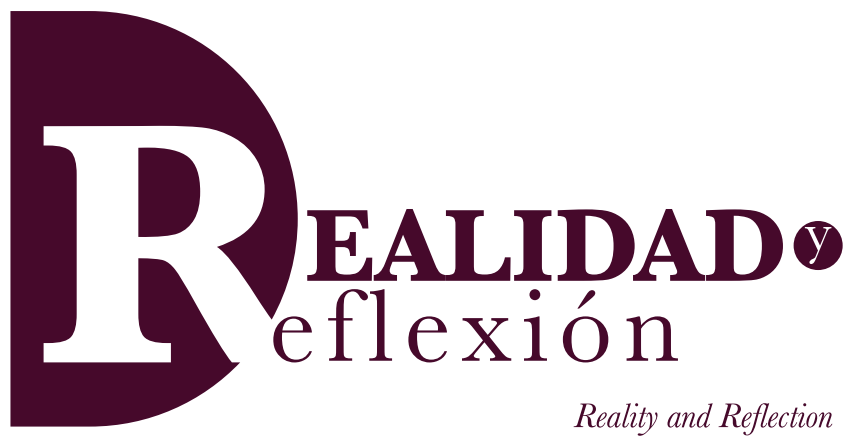

ISSN 1992-6510

Año 20, N 52, San Salvador, El Salvador, Centroamérica. Revista Semestral Julio-Diciembre 2020

YEAR 20, $N^{\circ}$ 52, SAN SALVADOR, El SALVADOR, CENTRAL AMERICA. SEMESTRAL JOURNAL JULY-DECEMBER 2020

\title{
La educación superior como campo de análisis de las políticas públicas: enfoques sobre el tema
}

\author{
Issues of public policy analysis based on a bigher \\ education approach
}

Dr. Herberth Alexander Oliva Docente e investigador de la Universidad de Guadalajara, México Investigador asociado Instituto de Ciencia Tecnología e Innovación de la Universidad Francisco Gavidia holiva@ufg.edu.sv

Dr. José Salvador Hernández Mayorga Consultor educativo para el área de políticas públicas y asesor legislativo pashaca71@gmail.com

Fecha de recibido: 13 de febrero de 2020 Fecha de aprobación: 31 de mayo de 2020 DOI: $10.5377 /$ ryr.v52i52.10641

(c)(1)(2) 


\section{RESUMEN}

Un punto de partida de profundizar en el conocimiento vinculante entre la educación superior y las políticas públicas es presentar en este escrito un análisis argumentativo que permita entender por qué la educación superior puede verse como un campo relevante de las políticas públicas, retomando para ello algunos enfoques de análisis y aportaciones de algunos especialistas en el tema. Se parte de la interrogante ¿Es la educación superior un campo de estudio de las políticas públicas?

El objeto de este trabajo es ofrecer, por lo que se refiere al sistema de educación superior, un intento de aclaración simplificado de la relación con la política pública, dejando clara la postura que los esfuerzos que hoy se realizan para lograr un ajuste vinculante entre educación superior y las políticas públicas, plantean con urgencia la necesidad de definir en términos claros y operativos, la forma en que ha de incorporarse la educación superior como campo de análisis político para integrarse en forma estimulante a los procesos de desarrollo y cambio.

Bajo el entramado constructo de ideas, se intenta sentar postura sobre como el desarrollo de la nación mexicana, por sus características y por su velocidad, ha contribuido a destacar, cada vez con mayor intensidad, el papel que la educación debe desempeñar en los procesos de cambio económico y social, cuando ajusta dinámicamente a sus demandas por cuanto en buena parte de los casos, las políticas educativas no suelen ser de manera concreta "buenas políticas de Estado", y terminan en algunos casos por ser propuestas dentro de las agendas de políticas públicas, que son sometidas a la alternancia y a los vaivenes personales de determinados actores, quienes buscan fragmentar una adecuada gestión educativa.

Palabras claves: educación superior, políticas públicas, análisis, enfoques.

\section{ABSTRACT}

A starting point that deepens the binding knowledge between higher education and public policies, is to present in this paper, an argumentative analysis that allows us to understand why higher education can be seen as a relevant field of public policies, returning to this some analysis approaches and contributions of some specialists in the subject. Be part of the question Is higher education a field of study of public policies?

The purpose of this work is to offer, as regards the higher education system, an attempt to simplify the relationship with public policy, making clear the position that the efforts made today to achieve a binding adjustment between education Higher and public policies, urgently raise the need to define in clear and operational terms, the way in which higher education has to be incorporated as a field of political analysis to integrate in a stimulating way to the processes of development and change.

Under the framework of constructing ideas, an attempt is made to establish a position on how the development 
of the Mexican nation, due to its characteristics and its speed, has contributed to highlighting, with increasing intensity, the role that education should play in the processes of economic and social change, when it dynamically adjusts to its demands because, in a good part of the cases, educational policies are not usually concretely "good State policies", and end up in some cases because they are proposed within the policy agendas public, who are subjected to the alternation and the personal fluctuations of certain actors, who seek to fragment an adequate educational management.

Key words: Higher Education, Public Policy, Analysis, Approaches. 


\section{A modo de introducción}

Un punto de partida que da pie para profundizar en el conocimiento vinculante entre la educación superior y las políticas públicas es presentar mediante un análisis argumentativo, un basamento teórico que permita entender por qué la educación superior puede verse como un campo relevante de las políticas públicas, retomando para ello algunos enfoques de análisis y aportaciones de algunos especialistas en el tema.

Bajo este marco introductorio se plantea el argumento que, dentro de las encrespadas y vertiginosas aguas del conocimiento, los sistemas educativos en el mundo demandan de un análisis permanente de las políticas públicas en el campo educativo a nivel superior, por medio del cual puedan tener un panorama de avanzada claro, con objetivos congruentes y que le permita a los académicosyespecialistas en el tema,empoderarse de manera convincente que el análisis de las políticas públicas educativas se vuelve imperante para interpretar e intervenir en mejor forma las grandes limitaciones y deficiencias de los sistemas educativos, y asumir que la mejora educativa urge de una reflexión que conduzca al trabajo incansable de todos los miembros de la comunidad educativa por alcanzar procesos de enseñanza eficientes y eficaces pensados en la canalización de aprendizajes significativos y en la formación de los perfiles profesionales que demanda la sociedad en la actualidad.

Desde un primer plano del análisis, es relevante considerar que la educación superior ha ido constituyéndose dentro del devenir de los tejidos sociales de las naciones, como uno de los principales instrumentos políticos con el que intentan (las naciones) garantizar el desarrollo y progreso de sus pueblos, por lo que, es importante asignarle un financiamiento digno a la educación superior para que esta pueda garantizar un buen nivel de calidad, con el fin de generar los resultados positivamente esperados en beneficio de las grandes mayorías sociales. De esta manera es como surge la interrogante que da vida al presente ensayo ¿Es la educación superior un campo de análisis de las políticas públicas?

En esa sintonía, se podría considerar que, si se analizan las políticas educativas a la luz de las políticas públicas debería partirse de la relación de dos elementos que se unifican mutuamente y que subyacen en la denominación de las políticas públicas, estamos refiriéndonos por un lado a la noción de lo público y un segundo punto al tema de la decisión. $Y$ es que la política pública implica que las estrategias de intervención y acción tengan un sentido público, sirvan a un interés público y/o respondan al resultado de la agregación de voluntades individuales del ciudadano, permitiendo que se debatan en público (las estrategias de intervención), y se asocien a la rendición de cuentas y al manejo transparente de los recursos. Dicho lo anterior y en la concreción de ideas que den el respectivo basamento epistémico a este aporte bibliográfico, encontramos a Picardo (2010) que considera que la reflexión sobre políticas públicas debe centrarse en las ideas o reflexiones, estratégicas que hagan posible lo necesario para la educación, rescatando para tal efecto la opinión del catedrático de Harvard, Gary Orfield, al señalar que "la política educativa orientada hacia la resolución de problemas concretos es de sustancial importancia para lograr avances significativos en educación". 
Para Picardo, es viable encontrar cómo en su argumento considera que siendo la educación la brújula de la globalización, con mayor razón habría que reflexionar sobre la orientación política del tipo de educación deseada; es decir, una educación de calidad, pertinente, eficiente y competitiva, que posibilite la inserción de los países en el fenómeno global.

\section{Ideas para el debate}

Las maneras y los estilos en cómo pensamos y diseñamos las políticas educativas ha cambiado en buena medida como resultado de las variadas modificaciones que se dan en los contextos sociales de las naciones alrededor del planeta. En casi todos, se han suscitado olas de democratización, modernización y cambio político y esto va definiendo los tipos de políticas que construimos. (De los Arcos, 1978, p. 219).

Lo anterior permite conjeturar que, en buena parte de los casos, las políticas educativas no suelen ser de manera concreta buenas políticas de Estado, terminan en algunos casos por ser propuestas dentro de las agendas de políticas públicas, que son sometidas a la alternancia y a los vaivenes personales de determinados actores, quienes buscan fragmentar una adecuada gestión educativa.

Algunos versados en el tema de políticas educativas como Flores (2015) afirman que:

En México, se suele considerar que hay un gran ojo conspirador en algunos organismos internacionales como el
Banco Mundial o la Organización para la Cooperación y el Desarrollo Económico (OCDE) que todo lo concerniente a políticas educativas se decide desde arriba; por lo que las cosas no son tan sencillas. ${ }^{1}$

Es interesante ver en el postulado del autor arriba mencionado, como el considerar que el desarrollo de un ejercicio de política educativa comparada, podría mostrar de manera clara y contundente que, las interacciones entre los políticos mexicanos y las élites internacionales son y siempre serán complejas, ya que siempre han existido recurrentemente a lo largo de la historia, además, evidencia que tanto México ha adoptado racionalmente políticas y programas educativos de otros países como también los ha transferido a muchas naciones, por tanto que, la educación superior si puede considerarse como un campo de las políticas públicas.

Al sostener esa relación dual entre educación superior y política pública, muchas pueden ser las experiencias que desde el contexto de la realidad concreta, evidencien como algunas instituciones de educación superior han sido utilizadas como instrumentos de manejo y dominio político, por lo que variados son los casos en los que las universidades sometidas a regímenes políticos, "se convierten en un poderoso instrumento de control para instrumentar reformas al interior de las instituciones de educación superior con el objetivo de adecuar las demandas del sector productivo al nuevo modelo de gestión pública" (Da Rocha Silva, 2008, p. 11).

1 Conferido de FLORES, Pedro (16 julio de 2015). Porque enseñar política pública. http://www.educacionfutura.org/por-que-ensenarpolitica-educativa/ (URL). 
Cuando se piensa en la educación superior como un campo de las políticas públicas, es viable retomar el argumento de Shepsle (2003) quien considera que "La política real involucra la negociación de acuerdos que tiene como núcleo el concepto de alineación de las expectativas con respecto a las obligaciones recíprocas $y$, en última instancia, a los resultados que las partes alineadas se proponen asegurarse". Dicho de otra manera, el estudiar las políticas educativas con un enfoque real y negociador, se ha ido consolidando y tomando nuevos bríos a lo largo de los últimos 10 primeros años del siglo XXI. Prueba de ello son las múltiples políticas educativas que al menos en territorio mexicano ayudan a instrumentalizar en la educación superior, una forma de garantizarle a sus estudiantes, un camino al crecimiento y desarrollo, para hacer frente a la revolución tecnológica y científica a la que se asiste en las últimas décadas en un contexto internacional cada vez más globalizado conforme a la dinámica propuesta por el modelo económico imperante que involucra la negociación de acuerdos, así como la alineación de las expectativas con respecto a las obligaciones recíprocas.

Buena parte del argumento académico centrado en la educación superior como campo de las políticas públicas, evidencia que muchos de los enfoques que albergan la teoría alrededor del tema, hablan de los principales retos que deben comenzar a afrontar las instituciones de educación superior, ya que al ser consideradas máximos exponentes de la formación y de la producción de conocimiento de una sociedad, necesariamente deben asumirse como actores protagónicos en el análisis de la política pública.
Sobre lo anterior, Aguilar (2009) argumenta que:

El centro de la disciplina y ejercicio profesional de las Políticas Públicas es la actividad intelectual del análisis de las políticas, con el fin de identificar las acciones que tendrían como efecto la ocurrencia de aquellas situaciones sociales que un gobierno y su sociedad desean realizar debido a su valor público o porque representan una aceptable solución de problemas y males públicos indeseados.

$\mathrm{Y}$ es que, dicho de otra manera, Aguilar intenta dejar en claro que el corazón de las políticas públicas ha consistido en producir y validar conceptos, hipótesis, modelos, métodos que permiten sustentar la corrección intelectual del análisis en servicio de las decisiones que toman los gobiernos para cumplir sus funciones públicas y dirigir a su sociedad, sobre todo cuando se trata de políticas educativas para el caso concerniente a este ensayo.

Aguilar considera que el análisis de las políticas públicas convierte los juicios de valor en juicios de hecho, ya que permiten desagregar los objetivos en resultados de acción, lo que se traduce en un conjunto limitado de realidades (observables, mensurables) que representan los resultados o efectos de las acciones de la política y la realización del objetivo que se persigue. Tal como se puede apreciar, el hibrido derivado de la política pública con la educación superior aterriza en lineamientos generales que desde la macro visión social deben integrar los aportes de la sociedad para crear una la política pública educativa objetiva y libre de sesgos. 
Acosta (2002), sociólogo y politólogo mexicano, dice que:

Las universidades son instituciones entendidas como espacios sociales que originan y regulan unos modos particulares de relación entre sujetos, actividades y puntos de vista; formas de poder y maneras de ejercerlo y disputarlo; modalidades diferenciadas y dictadas por la tradición, de acceder o no a los recursos, oportunidades y responsabilidades institucionales. Además las universidades están dentro del campo de la educación superior, campo que tiene sus propias lógicas de poder, y cuyos actores principales son (i) élites y grupos de poder social, económico y político dominantes; (ii) gobiernos y burocracia estatal; (iii) sectores estudiantiles y de docentes organizados y no hegemónicos; (iv) grupos sociales emergentes que buscan una educación universitaria rápida y no muy costosa como factor de movilidad; (v) comunidad académica y científica.

De esta manera, Acosta define que las políticas educativas son una construcción colectiva debido a la participación de actores que expresan las intencionalidades $\mathrm{y}$, en consecuencia, que la consecución de las metas y objetivos que se propone una política depende no sólo de la capacidad de gestión del gobierno y la burocracia, o de la capacidad de coacción del Estado, sino de la colaboración de las instituciones a quienes está dirigida y que las acatarían porque están convencidas de su pertinencia, oportunidad, efectividad y eficacia.
En síntesis, las políticas públicas en educación superior deben ser tomadas y desarrolladas por la sociedad civil, por los actores principales de la política y quienes serán beneficiados o afectados. Por lo que será el Gobierno de turno quien debe ser capaz de encauzar estas voces y requerimientos para que las políticas sean ante todo pertinentes y coherentes con la visión que se comparte entre los actores interesados. (Avendaño, W.R., Paz, L. y Vera, G. R., 2017)

En consecuencia, una clara postura que podría fortalecer el marco de discusión en el presente aporte, es la idea de asumir que el análisis de políticas educativas sí tiene cabida en un campo político, puesto que una política educativa adquiere un sentido valorativo de profundo impacto cuando se relaciona con los ideales de sociedad. Sumado a lo anterior, se debe considerar que es precisamente la legitimidad experiencial del proceso de elaborar políticas públicas, lo que contribuye que el hacer políticas educativas conlleve a la necesidad de hacer decisiones, y esto hace posible el construir un camino de acción y significado que involucra a quienes legítimamente están inmiscuidos o afectados.

Lo anterior es comprensible si se toma en consideración que, en todo momento de la existencia humana, se hace uso del análisis como un mecanismo que ayuda a comprender, en mejor medida, el medio social, político y económico en el que están inmersas las políticas educativas, por cuanto al considerarlas campo de análisis de la política pública, nos permite entender que no siempre son los politólogos (supuestos expertos en políticas educativas) los que elaboran o colaboran en el diseño de las políticas educativas; lo cual da paso a que en 
múltiples ocasiones sea fácil identificar cómo los politólogos se transforman en críticos, y no asesores en materia de política educativa.

Cuando se busca otorgarle legitimidad a la educación superior como campo de políticas públicas, vemos en el argumento de Klein un posicionamiento teórico que da soporte vital para comprender que la configuración de los sistemas educativos, se deben someter a procesos metodológicos de innovación, reordenamiento y cambio, los cuales son elementos vitales que deben pasar por instituciones flexibles con capacidad de dar respuesta a las modificaciones pertinentes. $\mathrm{Al}$ respecto Klein (2003) sostiene que:

Las organizaciones públicas deberían considerarse como sistemas de aprendizaje, que desarrollan su capacidad para adaptarse y responder a los cambios en un entorno que cambia rápidamente, valorando siempre que las instituciones se crean con el fin de cumplir ciertos objetivos y representar determinados valores que no pueden dejarse en manos del mercado.

Como cierre del apartado, diremos que al hacer un marco de comparación entre los argumentos vertidos por De los Arcos, Shepsle, Aguilar y Klein; estos nos conducen al punto medular que justifica que es precisamente la ausencia de la no contemplación de la educación superior como campo de las políticas públicas, un principio problematizador que deberá por tanto buscar la inclusión y equidad de directrices que den basamento a que las políticas educativas se retomen a partir del resultado de la exposición y análisis de múltiples argumentos, acuerdos y los consensos en beneficio de las grandes mayorías sociales.

\section{Argumentos, posturas y enfoques de análisis}

Como punto de partida en este segundo apartado, se debe agregar al conglomerado de ideas que, la cuestión del cómo se relaciona la política con las políticas públicas es uno de los grandes puntos de conexión que validan a la educación superior como campo de análisis político; es que, se debe entender a la política como uno de los elementos fundamentales que sirven de eje de acción del gobierno, llevándonos al punto de reflexión relacional que cuestiona ¿de dónde surge el estudio de las políticas públicas como parte de la solución a las problemáticas del pueblo? Y es que existe una preocupación por las relaciones complejas y poco obvias entre politics y public policies como expresión o concepto que acuñó el economista Petter Evans en los años 70 para señalar el déficit de comprensión de políticas públicas.

Evans en su texto La gobernanza de la economía planteaba una división artificial que dejaba en evidencia que la política no tenía, en un inicio, nada que ver con la política pública, donde la política era el ámbito de la negociación o conflicto y las políticas públicas eran el ámbito de la ejecución de las acciones. Por lo cual, sí es viable relacionar la idea de que toda política pública es una decisión política. En este marco de análisis, se concluye que la idea de que las políticas públicas y gubernamentales obedecen a principios de decisión política está más orientadas a resolver conflictos de carácter político, lo cual coloca al frente la postura de saber hasta donde es política o hasta donde es política pública.

Dicho de otra forma, el párrafo anterior propone mirar en perspectivala relación entre instituciones 
políticas, procesos de políticas y resultado de políticas; donde el tipo de instituciones políticas definen el tipo de procesos y resultados políticos, dejando en claro, además, que existe evidencia empírica que pone en evidencia dicha relación. Por lo tanto, es relevante considerar que uno de los primeros autores que proponen una visión esclarecedora entre la relación de política con políticas públicas la presenta Robert Dahl en su libro Quién gobierna, donde se argumentaba que un punto central en la discusión es la cuestión de saber si "los gobiernos saben lo que hacen".

Por otro lado, vemos de manera más clara y directa en el planteamiento de Giliberto Capano un argumento solido que justifica por qué la educación superior debe verse como un campo de políticas públicas, al respecto Capano (1996) sentenciaba que "las universidades, al gozar de autonomía, y al contener comunidades que hacen políticas públicas para sí y para otros, otorgan la principal característica para ser estudiadas como un privilegiado campo de investigación”.

La versatilidad del análisis planteado por el autor permite compartir una idea en la cual el cambio político o cambio institucional es un objeto de estudio relevante en las ciencias sociales y, por ende, en la educación superior. Para tal efecto retoma 3 nociones:

- La primera plantea que la investigación en política no solo es para describir el poder, sino también las soluciones a problemas colectivos.

- En la segunda noción, Capano comparte una idea de cómo el cambio político permite configurar modelos para explicar la relación entre factores estructurales e individuales.
- Y una tercera noción, permite conocer el concepto de red como arsenal (lentes o filtros) útiles para analizar las relaciones.

Capano busca de esta manera, poder otorgarle un especial énfasis a lo significativo de explicar que la educación superior forma parte latente del análisis político, y como este análisis político forma parte a su vez del diverso y complejo cambio político; por lo que el propósito que busca tan celebre intelectual es el de complementar los enfoques que analizan los procesos de política pública en educación superior y la lógica de los actores involucrados, y cómo los microcomportamientos dan forma y restringen las elecciones de política a aplicar cuando se trata del campo educativo.

Un análisis exhaustivo de lo anterior permite poner pesos y contrapesos en la balanza analítica, y plantear la idea contraria al punto medular del argumento manejado en este ensayo, derivándose de ello el apartado que señala por qué no se debe de considerar a la educación superior como campo de análisis de las políticas públicas; en la medida que por medio de las políticas podemos elaborar una reflexión minuciosa de políticas educativas del nivel superior, en donde se busca cuestionar si realmente los gobiernos ven algún potencial en las universidades como instituciones que colaboran con los tejidos sociales para afrontar, resolver y solventar problemas sociales definidos como problemas públicos, justificando nuevamente una idea que defienda que la educación superior si puede ser campo de acción de la política pública.

No se debe de perder de vista que la educación superior entendida como un campo de políticas, permite ver distintos acercamientos a la 
educación superior como un campo de acción pública en el cual se intenta caracterizar a la política de educación como política pública. No obstante, se deben considerar para el caso, dos maneras de entrar en este campo; la primera es usar el método comparativo, el cual supone un ejercicio que permita distinguir las variables claves de un campo de políticas de educación, y por otro lado ,se encuentra el método de estudio de casos. Ambos métodos tienen sus ventajas, encontrando en el método comparativo la ventaja de que sus conclusiones son generalizadas por lo que las fortalezas de las variables elegidas permiten elaborar más estudios; ello implica multiplicar las variables en pocos casos. En el caso del método de estudio de casos, se considera una ventaja el poder identificar pocas variables identificando el número de casos.

Una de las grandes aportaciones que hace Capano al campo teórico y metodológico de las políticas educativas, es determinar que las universidades, al gozar de autonomía, y al contener comunidades que hacen políticas públicas para sí y para otros, dan lugar para ser estudiadas como un privilegiado campo de investigación política. El célebre académico considera para ello que, el cambio político o cambio institucional es un objeto de estudio relevante en las ciencias sociales y, por ende, en la educación superior Capano advierte con suspicacia que la investigación en política no solo es para describir el poder, sino también para describir las soluciones a problemas colectivos, por lo que la explicación del cambio político permite configurar modelos para fundamentar la relación entre factores estructurales e individuales donde se visualiza el concepto de red como arsenal (lentes o filtros) útiles para analizar las relaciones.
El autor da énfasis en lo significativo de explicar el diverso y complejo cambio político y cuan deficiente es esa explicación cuando se basa en modelos que consideran solo factores macro. $Y$ es que para Capano, el propósito es complementar los enfoques macro que analizan los procesos de política pública en educación superior y la lógica de los actores involucrados, y como los micro comportamientos dan forma y restringen las elecciones de política a aplicar.

Para dar un buen basamento al soporte de ideas que comparte Capano, retoma a Kogan (1984) al puntualizar que, en la educación superior, probablemente mejor que en cualquier otro campo, es factible estudiar cómo lo público tiene sus modos de operarse en lo privado. Retoma además desde el enfoque metodológico que el análisis enfocado en las políticas y el cambio que se produce como objeto de investigación dentro de la educación superior, forma parte de una estrategia comparativa de investigación (casos institucionales o experiencias nacionales) que dejan en evidencia que las políticas públicas y los cambios que producen en el tiempo, generan un conocimiento que clarifica el por qué la educación superior es parte del análisis del campo de las políticas y cita a (Sabater, 1991).

Para cerrar el apartado referente a Capano sugiere 3 niveles de análisis (Clark 1983), desde los cuales la dimensión política en la educación superior se plasma en los distintos caminos que puede tomar, para ello menciona a las unidades Básicas (facultades o departamentos), señala además a la Institución de Educación Superior (la universidad analizada), retoma también a las autoridades centrales (gobierno, congreso, burocracias, órganos centrales de coordinación) 
y concluye con el argumento que debemos entender la interdependencia o los roles, sin caer en un mare magnum de datos.

Otro importante autor que se abre campo en el análisis de la educación superior, como campo de las políticas públicas, es Ladislav Cerych, quien propone que un análisis de implementación de políticas públicas puede comenzar por considerar como objetivos de políticas (para el casolas políticas educativas) aquellos formalmente adoptados por parlamentos o gobiernos, lo cual genera el cuestionamiento de si los objetivos de las políticas suelen ser un factor importante en el proceso de implementación (para el caso las políticas públicas), tomando en cuenta que el análisis de implementación tiene su complejidad, haciendo evidente que ello (el análisis de implementación) se vuelve una situación donde existe una multitud de actores. $Y$ es que cuando se trata analizar con detenimiento la implementación de políticas, pero particularmente las políticas educativas, se debe partir del diseño, definición, su adopción, su posterior reformulación, y su conversión hacia una práctica de rutina o de rechazo.

Por consiguiente, Cerych sitúa su argumento en la idea que, al evaluar la política pública, es posible realizar una medición de los resultados encontrados en el proceso de indagación que ayuda a comprender si una política tuvo éxito o no lo tuvo. Ello permite entender como el diseño de las políticas públicas educativas deben hacerse dentro de una conjetura racional que potencie la mejor forma de tomar decisiones y acciones contenidas en una agenda de las políticas públicas.

Asimismo, es relevante distinguir que, al evaluar las políticas educativas, ayuda a enfocarnos en los procesos más que en los resultados mismos, obteniendo de esa manera un mecanismo para cuantificar el logro de cumplimiento de dichos objetivos. Y es que la relación de evaluación con el diseño de las políticas públicas nos encamina al encuentro de las causas que motivan o impiden el propósito final de las políticas públicas como una respuesta a las diversas problemáticas complejas y diversas en el plano educativo.

Por lo tanto, dentro del análisis de políticas públicas de Cerych (1974), considera relevante destacar desde cuatro apartados que se detallan a continuación:

- Menciona en primer lugar a la naturaleza del análisis de políticas y su relación con otras perspectivas de las ciencias sociales,

- Habla principalmente de un análisis interesado en causas, contenido y consecuencias de las políticas públicas,

- Considera que un análisis de políticas públicas debe poseer una valoración del grado en que los objetivos han sido cumplidos, dando para ello las razones que lo justifican, además de los argumentos que validen la distorsión de los objetivos originales, así como la evaluación de los efectos involuntarios de ciertas políticas,

- Por último Cerych habla de un análisis de políticas públicas a partir delaimplementación, el cual se ocupa de los procesos, en lugar de medir el impacto de la política.

Tanto Capano como Cerych, adoptan posturas comparatistas en el análisis de políticas públicas en la educación superior, ambos plantean algunas 
ideas fundamentales del porque en algunos países triunfan las reformas y en otros no funcionan las reformas educativas.

Por un lado, Capano propone un argumento de cómo se traducen las políticas públicas en propuestas de análisis de las políticas, es decir, propone herramientas para entender en mejor forma cómo la política pública se transforma en educación superior.

Cerych, por su parte, comparte una idea mediante la cual se identifica el proceso de expansión de la educación superior a través de la elección de las políticas de diversificación de la oferta de la educación superior. Se podría interpretar basado en el argumento anterior que es viable proponer una idea de que la educación ayuda a la mejora de la productividad de los países, considerando que la educación ayuda a los pueblos a tomar las decisiones e implementar dichas decisiones derivadas de las políticas públicas, teniendo de esa manera una evidencia tangible de lo que dejan las políticas públicas en función de sus objetivos.

Por lo tanto y como parte del sostenimiento del argumento antes planteado, se considera viable asumir al gobierno como un administrador de las políticas públicas, donde este gobierno aparece como una caja negra que en muchas ocasiones no afronta el objeto de estudio de la política misma, aun y cuando sabiendo que, desde el derecho, la economía, la administración y la política propiamente dicho (4 patas fundamentales de la política) se intenta abordar un objeto político, logrando apuntalar que al final los gobiernos no saben lo que hacen. Esto da paso a entender como el Estado, al ser un financista parcial de la educación superior pública, se vuelve un personaje multifacético al transformarse en un claro proveedor de préstamos a los estudiantes; un excelente promotor del acceso educativo a través de becas, un regulador del mercado educativo a través de mecanismos de aseguramiento de la calidad y proveedor de incentivos en función de objetivos y prioridades para el desarrollo del sistema nacional de educación superior.

Cada vez retoma fuerza la perspectiva intelectual que la educación superior da vida en buena parte al análisis de la política pública, ya académicos de la talla de Nicholas Barr (2005) consideraban que:

Existen beneficios que la educación superior produce para la sociedad en su conjunto y que van más allá de los beneficios individuales o privados, tales como el crecimiento económico, la cohesión social, una transmisión de valores y un desarrollo del conocimiento como un fin en su propio mérito.

$Y$ es que, al reflexionar en el argumento emitido por Barr, viene a la mente la idea que todas transformaciones por las que atraviesa cualquier sistema educativo en el mundo van a requerir siempre de reformas y políticas educativas enfocadas en potenciar la mejora y el aumento en la calidad de la educación, y que a la vez se genere un proceso de transformación del sistema educativo mediado por políticas del estado que propicie el bienestar de todos los colectivos.

No obstante, se debe señalar que, en los países más desarrollados del mundo, es fácil percibir cómo la educación superior forma parte elemental del análisis de las políticas públicas, al 
asignarle a toda política educativa un rol de vital importancia que justifique pertinencia, eficiencia y eficacia de todas las acciones que impliquen la mejora constante de todos los elementos que contribuyen a los sistemas educativos. Desde esta perspectiva, nos encontramos con suficientes indicios de esta aseveración; en este sentido encontramos que una política educativa orientada hacia la resolución de problemas concretos es de sustancial importancia para lograr avances significativos en educación.

En la valiosa opinión de expertos en el tema como Navarro (2006) se afirma que:

Para entender por qué el cambio en política educativa es difícil, hay que comprender a los actores principales, sus preferencias y horizontes temporales, cómo se alinean sus intereses y, por ende, las posibilidades de conflicto o cooperación, así como los entornos en que se formula la política.

Lo previamente citado indica que los cambios en educación tienen su nivel de dificultad, pero en la medida que se estudia a los actores del proceso se puede lograr cooperación en vez de conflicto con sus agentes protagónicos. Por lo cual, los cambios en educación son políticamente factibles siempre que contengan un horizonte de transcendencia que va más allá de lo simple, ya que llega a todos los miembros de una forma $\mathrm{u}$ otra obtienen beneficios del sistema educativo.

\section{Conclusiones}

Mientras las políticas educativas sigan siendo entendidas como un ejercicio del poder que busca canalizar resultados deseados en el sector educativo, se deberá intentar hacer todo lo posible para gestionar decisiones, planes, programas y acciones concretas que materialicen la eficiencia y eficacia del hecho educativo. En virtud de ello se proponen algunos argumentos concluyentes que se detallan a continuación:

a. La educación superior demanda de un análisis político cuyos insumos estratégicos den cabida a la intervención en el sector educativo mediante el curso de acción y diseño efectivo de las políticas educativas y su idónea implementación.

b. Se debe considerar que, siendo la educación un camino latente que marca la ruta hacia la globalización y la modernización, se debe dar más valor a la acción reflexiva de la pertinencia de la educación superior como campo de análisis de la política, ya que en esa medida podrá surgir una amplia y marcada orientación sobre el tipo de educación superior deseada, es decir que, en la búsqueda de una educación de calidad, pertinente, eficiente y competitiva, se vuelva posible una inserción de las naciones en el fenómeno global de la sociedad del conocimiento que se apoye en la educación superior como un elemento activo de razonamiento político.

c. De esta forma, con el abordaje temático del presente escrito, se intentó crear un enfoque que facilitará la comprensión de la educación superior como campo de análisis de la política pública, dejando ver que la configuración de políticas educativas forma parte fundamental de la acción política. Es por tanto, inevitable considerar que dentro de la estructura de 
una educación superior aparecen múltiples y complejos actores que desde variados elementos de análisis como la realidad social, una visión permanente de mejora continua, y una visión de futuro centrada en metas concretas, se puede generar un debate y discusión alrededor del campo de las políticas públicas.

d. No es posible pensar en un vínculo entre educación superior y política pública sin considerar que no puede existir una política educativa sin datos o sin información pertinente y ágil que ayude a trascender al campo ideológico político del cual pueda surgir una concepción de poder consultado, consensuado y con disensos administrados para el diseño mismo de la política educativa.

En resumen, la política pública de educación superior parte de la inversión pública y la sostenibilidad financiera para la obtención del éxito en su dinámica funcional, por lo que el aspecto de una formulación de la agenda política permite que se generen escenarios que contribuyan a encauzar y a negociar adecuadamente los conflictos y los intereses que puedan ser contradictorios en la dinámica funcional del sistema educativo, dándole con ello a la educación superior un alto valor político.

En último lugar, tanto la educación superior como las políticas públicas se caracterizan por poseer de elementos de mejora frente a las situaciones que afectan a los sectores educativos, por lo que, los gobiernos, deberán impulsar reformas educativas que favorezcan al desarrollo del sistema, considerando evaluaciones, sugerencias y metas que aporten al proceso de transformación educativa de manera significativa facilitando medios y recursos para propiciar el diálogo, el consenso y acuerdos que influyen directamente en el logro de los objetivos y metas de los sistemas educativos.

En concreto, el actor principal, autorizado y legitimado para velar por la ausencia de obstáculos en la implementación de la educación superior debe ser el Estado, el cual debe utilizar su poder político como herramienta que permita la adecuada conducción de una aplicación fluida de la política pública en ámbitos educativos.

\section{Bibliografía}

Acosta Silva, A. (2002). El neointervencionismo estatal en la educación superior en América Latina. Revista Sociológica. Año 17, número 49, mayo-agosto, páginas 43-72.

Acosta Silva, A. (2000). Estado, politicas y universidades en un periodo de transición. México: FCE y Universidad de Guadalajara.

Aguilar Villanueva, L. (2009). Marco para el análisis de políticas públicas. Administración y ciudadanía, 3 (2), 1-28.

Barr, N., and Crawford I. (2005). Financing Higher Education Answers from the UK. Recuperado en: https://www.researchgate.net/ publication/44835507_Financing_Higher_ Education_Answers_from_the_UK

Bovens, M., Hart, P. y Kuipers, S. (2008). The politics of policy evaluation. In Moran, M., Rein, M. $y$ Godin, R. The oxford handbook of public policy. New York: Oxford University Press. 319-335. 
Capano, G. (1996). Political Science and the Comparative Study of Policy Change in Higher Education. Theorético - Metodological Notes From a Policy Perspective. Higher Education, 31(3), 263-282.

Cerych, L. (1974). The Policy Perspective. Clark, B. R. (Ed.), Perspectives on Higher Education: Eight Disciplinary and Comparative Views [en línea] (pp. 233-255). Berkeley: University of California Press.

Delos Arcos, R. (1978). Introducción al pensamiento politico y nociones gubernamentales, pag. 219 - 277. Editorial MOSSE, Santiago de Chile.

Majone, G. (2008). Agenda setting. In Moran, M., Rein, M. y Godin, R. The oxford handbook of public policy. Reino Unido: Oxford University Press.

March, J. y Olsen, J. (2008). The logic of appropriateness. In Moran, M., Rein, M. y Godin, R. The Oxford Handbook of public policy. Reino Unido: Oxford University Press.

Navarro, J. (2006). Dos clases de políticas educativas. La política de las políticas públicas. Buenos Aires, Argentina. Editorial PREAL No 36. Recuperado en: https://ubc.edu.mx/ plataformavirtual/pluginfile.php/15009/ mod_resource/content/1/Texto\%201.\%20 Dos\%20clases\%20de\%20pol\%C3\%ADticas\%20 educativas.\%20La\%20pol\%C3\%ADtica\%20 d e $\% 201$ a s $\% 20$ p o $1 \%$ C $3 \%$ A D t i cas $\% 20$ p\%C3\%BAblicas.PDF (10/10/2016)

Picardo, O. (2012) Políticas públicas educativas: Ensayos para el debate. San Salvador El Salvador. UFG Editores
Rhodes, R. (2008). Policy network análisis. In Moran, M., Rein, M. y Godin, R. The oxford handbook of public policy. New York: Oxford University Press. 425-447.

Rudolf, K. (2003)., Las instituciones adaptables: el diseño institucional y el estado de bienestar en Reino Unido. Teoría del diseño institucional / coord. por Robert E. Goodin, ISBN 84-7432852-7.

Shepsle, K. A. 1978. The Giant Jigsaw Puzzle. Chicago: University of Chicago Press.

Shepsle, K A. 1979. Institutional arrangements and equilibrium in multidimensional voting models. American Journal of Political Science, \# 23, pp. 27 - 59he Giant Jigsaw Puzzle. Chicago: University of Chicago Press.

Shepsle, Kenneth A. 1991. Discretion, institutions and the problem of government commitment, en Pierre Bordieu y James Coleman (comps), Social Theory for a Changing Society. New York: Russel Sage.

Silva, M. (2008). Políticas públicas para la educación superior: la implementación de la evaluación en Brasil y México. Perfiles educativos, 30(122), 7-37.

William Rodrigo Avendaño Castro, Luisa Paz Montes, Gerson Rueda Vera. Políticas públicas y educación superior: análisis conceptual del contexto colombiano. Revista Venezolana de Gerencia Universidad del Zulia, vol. 22, núm. 79, 2017. 